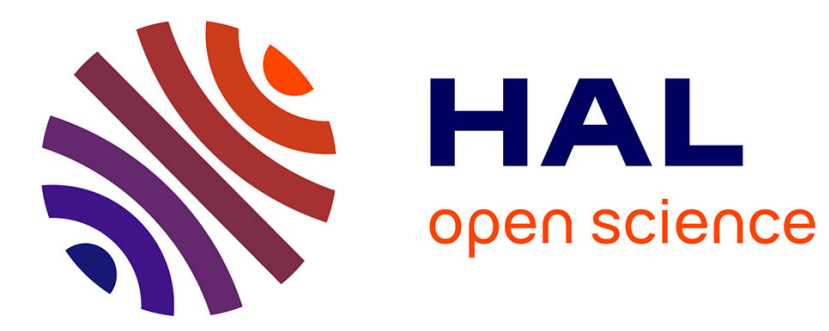

\title{
Human group X secreted phospholipase A 2 induces dendritic cell maturation through lipoprotein-dependent and -independent mechanisms
}

\author{
Rajai Atout, Sonia-Athina Karabina, Sandra Dollet, Martine Carreras,
} Christine Payré, Patrice André, Gérard Lambeau, Vincent Lotteau, Ewa Ninio, Laure Perrin-Cocon

\section{To cite this version:}

Rajai Atout, Sonia-Athina Karabina, Sandra Dollet, Martine Carreras, Christine Payré, et al.. Human group X secreted phospholipase A 2 induces dendritic cell maturation through lipoprotein-dependent and -independent mechanisms. Atherosclerosis, 2012, 222 (2), pp.367-74. 10.1016/j.atherosclerosis.2012.03.014 . inserm-02443429

HAL Id: inserm-02443429 https://www.hal.inserm.fr/inserm-02443429

Submitted on 17 Jan 2020

HAL is a multi-disciplinary open access archive for the deposit and dissemination of scientific research documents, whether they are published or not. The documents may come from teaching and research institutions in France or abroad, or from public or private research centers.
L'archive ouverte pluridisciplinaire HAL, est destinée au dépôt et à la diffusion de documents scientifiques de niveau recherche, publiés ou non, émanant des établissements d'enseignement et de recherche français ou étrangers, des laboratoires publics ou privés. 
Human group $\mathrm{X}$ secreted phospholipase $\mathrm{A}_{2}$ induces dendritic cell maturation through lipoprotein-dependent and -independent mechanisms.

Rajai Atout $^{\mathrm{a}}$, Sonia-Athina Karabina ${ }^{\mathrm{a}, \mathrm{b}}$, Sandra Dollet ${ }^{\mathrm{c}, \mathrm{d}}$, Martine Carreras $^{\mathrm{c}, \mathrm{d}}$, Christine Payrée, Patrice André $^{\mathrm{c}, \mathrm{d}, \mathrm{f}}$, Gérard Lambeau ${ }^{\mathrm{e}}$, Vincent Lotteau ${ }^{\mathrm{c}, \mathrm{d}, \mathrm{f}}$, Ewa Ninio ${ }^{\mathrm{a}}$, Laure Perrin-Cocon ${ }^{\mathrm{c}, \mathrm{d}, *}$.

a INSERM UMRS 937, Université Pierre et Marie Curie UPMC-Paris 6, Faculté de Médecine Pierre et Marie Curie, 91 Boulevard de l'Hôpital, Paris 75634 Cedex 13, France;

${ }^{\text {b }}$ Current address: INSERM UMRS 933; Université Pierre et Marie Curie UPMC-Paris 6, Hôpital Armand-Trousseau, 26 Avenue Docteur Arnold Netter, 75571 Paris Cedex 12, France;

${ }^{\mathbf{c}}$ Université de Lyon, France;

d INSERM, U851, 21 Avenue Tony Garnier, Lyon, F-69007, France;

${ }^{\mathbf{e}}$ Institut de Pharmacologie Moléculaire et Cellulaire, UMR 7275, CNRS et Université de Nice-Sophia Antipolis, 06560 Valbonne, France;

${ }^{\mathbf{f}}$ Hospices Civils de Lyon, Laboratoire de virologie, Hôpital de la Croix Rousse, France.

*Corresponding author:

Laure Perrin-Cocon

INSERM U851, 21 av. Tony Garnier, 69007 Lyon, France

Tel: $+33-437282447$

Fax: $+33-437282341$

Email: laure.perrin@inserm.fr 


\section{Abstract}

Objective: Increased secreted phospholipase $\mathrm{A}_{2}\left(\mathrm{sPLA}_{2}\right)$ activity has been documented in several inflammatory disorders. Among $\mathrm{sPLA}_{2} \mathrm{~s}$, the human group $\mathrm{X}$ (hGX)-sPLA 2 has the highest catalytic activity towards phosphatidylcholine (PC), the major phospholipid of cell membranes and blood lipoproteins. hGX-sPLA 2 has been detected in human atherosclerotic lesions, indicating that $\mathrm{SPLA}_{2} \mathrm{~S}$ are an important link between lipids and inflammation, both involved in atherosclerosis. The presence of dendritic cells (DC), the most potent antigen presenting cells, in atherosclerotic lesions has raised the question about their role in disease progression.

Methods and results: In this study, we show that hGX-sPLA 2 -treated LDL induces human monocyte-derived DC maturation, resulting in a characteristic mature DC phenotype and enhanced DC ability to activate IFN $\gamma$ secretion from T cells. hGX-sPLA 2 phospholipolysis of LDL produce high levels of lipid mediators, such as lysophosphatidylcholine (LPC) and free fatty acids (FFAs), which also modulate DC maturation. The major molecular species of LPC containing a palmitic or stearic acid esterified in the $s n-1$ position induce DC maturation, whereas the FFAs can positively or negatively modulate DC maturation depending on their nature. hGX-sPLA 2 added alone can also activate DC in vitro through the hydrolysis of the DC membrane phospholipids leading, however, to a different cytokine profile secretion pattern than the one observed with hGX-sPLA 2 -phospholipolysed LDL.

Conclusion: hGX-sPLA 2 secreted in inflamed tissues can contribute to local DC maturation, resulting in pro-Th1 cells, through the production of various lipid mediators from hydrolysis of either LDL and/or cell plasma membrane.

\section{Keywords:}


Phospholipolyzed LDL; hGX-sPLA 2; Lipid mediators; Dendritic cells; Immune response; Inflammation; Atherosclerosis. 


\section{Introduction}

Atherosclerosis is a chronic disease of the arterial wall in which inflammation and immunity interact to define plaque evolution [1]. In this process lipids and lipoproteins are known to play a key role. Among the immune cells detected in atherosclerotic plaques, dendritic cells (DCs) and T helper 1 (Th1) lymphocytes producing IFN $\gamma$ have been shown to participate in disease progression [2]. DCs which are specialized antigen presenting cells need to be activated, in order to efficiently stimulate naïve $\mathrm{T}$ cells and induce their differentiation into polarized effector cells. This polarization depends on the signals T cells receive from DCs by cell to cell contact and by soluble secreted mediators such as cytokines and chemokines. DC activation can be triggered by various signals, including pathogen-associated molecular patterns and endogenous alarm signals associated with tissue damage and/or inflammation. We have previously shown that oxidized low density lipoprotein (oxLDL), a key player in atherosclerosis can promote mature DC generation in vitro [3], mainly through the production of the immunostimulating molecule lysophosphatidylcholine (LPC) $[4,5]$. This suggests that oxLDL plays an active role in the interface between immunity and inflammation. Native LDL prevents DC maturation induced by oxLDL or LPC, suggesting that the balance between native and oxidized LDL regulates the activation threshold of DC [3]. Moreover, native LDL and HDL inhibit the functional maturation of DCs triggered by some toll-like receptor (TLR) stimulations [6]. These observations underline the role of lipoproteins and lipids in the modulation of the immune system indicating that biochemical modifications of lipoproteins during pathological conditions may reflect an abnormal situation and provide alarm signals to DCs, regulating their immune function.

Secreted phospholipases $\mathrm{A}_{2}\left(\mathrm{sPLA}_{2} \mathrm{~s}\right)$ can also be considered as endogenous alarm signals since their secretion is enhanced in inflamed tissues [7]. $\operatorname{sPLA}_{2} \mathrm{~s}$ constitute a family of structurally-related, disulfide-rich, calcium-dependent enzymes, which hydrolyze 
glycerophospholipids to produce LPC and free fatty acids (FFAs) [8]. These enzymes exhibit major differences in their catalytic activities, substrate preferences and receptor binding properties [8]. We and others have shown that human group IIA, III and V sPLA $2 \mathrm{~S}$ can induce or regulate DC maturation and function [9-11]. Furthermore, group IIA, III, V and X sPLA $2 \mathrm{~S}$ have been detected in human and/or mouse atherosclerotic lesions [12-14]. Among the sPLA $_{2} \mathrm{~s}$, the human group $\mathrm{X}$ sPLA $2\left(\mathrm{hGX}-\mathrm{sPLA}_{2}\right)$ has unique enzymatic properties and binds with high affinity to zwitterionic phospholipids, especially to phosphatidylcholine (PC), the major phospholipid of cell membranes and lipoproteins [15]. Hydrolysis of PC on LDL by hGX-sPLA 2 preferentially releases arachidonic or oleic acid and LPC [16]. hGX-sPLA 2 is produced as a proenzyme and its activation is likely to be tightly controlled in different cells and various physiopathological conditions $[7,17]$.

We previously showed that LDL phospholipolyzed by hGX-sPLA 2 (LDL-X) has a smaller diameter than LDL. LDL-X induces foam cell formation when added to human monocytederived macrophages [12] and initiates endothelial cell perturbation through ER stress signaling, providing an LDL oxidation-independent mechanism for the initiation of vascular inflammation in atherosclerosis $[18,19]$. Given the high activity of hGX-sPLA 2 on PC present in cell membranes and lipoproteins and its presence in the atherosclerotic lesions, along with LDL and DCs, we undertook the present study to examine the impact of phospholipolyzed LDL on DC maturation in vitro. 


\section{Materials and Methods}

\section{LDL isolation and treatment with hGX-sPLA}

LDL $(\mathrm{d}=1.019-1.063 \mathrm{~g} / \mathrm{ml})$ was isolated from frozen plasma by density gradient ultracentrifugation and was free of HDL [12]. Its protein content was determined by the bicinchoninic acid (BCA) method (Thermo Fisher Scientific, France). Freshly prepared filtered $(0.45 \mu \mathrm{m})$ sterile $\mathrm{LDL}, 1 \mathrm{mg} / \mathrm{mL}$ protein in buffer containing $1 \mathrm{mM} \mathrm{CaCl} 2,12.5 \mathrm{mM}$ Tris- $\mathrm{HCl}(\mathrm{pH}=8.0), 0.25 \mathrm{M} \mathrm{NaCl}$ and $0.0125 \%$ BSA was incubated with $100 \mathrm{nM}, 200 \mathrm{nM}$, or $500 \mathrm{nM}$ recombinant $\mathrm{hGX}-\mathrm{SPLA}_{2}$ [20] for $3 \mathrm{~h}$ at $37^{\circ} \mathrm{C}$ or $24 \mathrm{~h}$ at room temperature (RT) and denoted as LDL-X. LDL treated as above but in the absence of hGX-sPLA 2 is denoted as LDL. In selected experiments, the activity of hGX-sPLA 2 was blocked by $10 \mu \mathrm{M}$ sPLA 2 specific inhibitor LY329722. Conjugated dienes, a typical index of oxidation, were measured at $234 \mathrm{~nm}$.

\section{Non-esterified fatty acid (NEFA) and LPC measurement}

The extend of LDL hydrolysis by hGX-sPLA 2 was determined in an aliquot of hGX-sPLA $2_{2}$ treated and mock-treated-LDL using the NEFA-FS kit (Diasys, France) for NEFA quantification and the AZWELL LPC kit (Cosmobio, Japan) for LPC production.

\section{SDS-PAGE and Western Blot analysis}

5-20 $\mu \mathrm{g}$ of LDL or LDL-X was subjected to 7\% SDS-PAGE electrophoresis followed by either Coomassie Blue staining or Western Blot analysis with an apoB antibody (clone 1D1) from the Heart Institute (University of Ottawa, Ontario, Canada).

\section{Sudan Red staining of modified lipoproteins}

$20 \mu \mathrm{g}$ of LDL or LDL-X was subjected to $1 \%$ agarose gel electrophoresis followed by Sudan Red staining.

hGX-sPLA 2 activity measurement 
hGX-sPLA 2 activity was measured as previously described [21] using $50 \mu \mathrm{M}$ of $\left[{ }^{3} \mathrm{H}\right.$-acetyl]PAF final concentration. The effect of LPC and arachidonic acid (AA) on hGX-sPLA 2 activity was tested in the presence of $1,5,10 \mathrm{mg} / \mathrm{ml} \mathrm{AA}$ or LPC or both or equal volume of ethanol (ETOH), which was used as a vehicle for AA and LPC in a final reaction volume of $200 \mu \mathrm{L}$. The results were expressed as nmol of PAF degraded per min per ml.

\section{Preparation and treatment of monocyte-derived DC}

Monocytes were purified from human peripheral blood as previously described [3]. Purified monocytes $\left(10^{6}\right.$ cells $\left./ \mathrm{ml}\right)$ were differentiated to immature DCs with 50ng/ml human GM-CSF and $62.5 \mathrm{ng} / \mathrm{ml}$ human IL-4 (AbCys, France) for 6 days.

At day 5, differentiated DCs were incubated in complete RPMI 1640 medium supplemented with $10 \%$ lipoprotein-deficient fetal calf serum (LPDS, Sigma) containing $24.9 \mathrm{mg} / \mathrm{ml}$ total serum proteins, including albumin, and treated for $24 \mathrm{~h}$ with $50 \mu \mathrm{g} / \mathrm{ml}$ LDL or LDL-X (corresponding to a $20 \mathrm{x}$ dilution of the $1 \mathrm{mg} / \mathrm{ml}$ solution described above). The impact of hGX-sPLA 2 alone on DCs was evaluated by the addition of the same amount of hGX-sPLA 2 in DC culture as the amount of enzyme brought by LDL-X. In selected experiments, $2.5 \mu \mathrm{M}$ sPLA 2 specific inhibitor LY329722 was introduced in DC culture, 15 min prior to hGX$\mathrm{sPLA}_{2}$ or LDL-X treatment. Control cells were incubated with equal volume of LDL-X buffer. In separate experiments, DCs were treated with lipids solubilized in ETOH and kept under nitrogen at $-20^{\circ} \mathrm{C}$. Egg LPC or synthetic LPC (LPC16:0, LPC18:0, LPC18:1) were from Avanti polar lipids (AL, USA). Palmitic (C16:0), stearic (C18:0), oleic (C18:1) and arachidonic (C20:4) acids were from Sigma. They were added to DC cultures in small volumes of $\mathrm{ETOH}$ in the presence or the absence of LDL $(50 \mu \mathrm{g} / \mathrm{ml})$. Control cells received equal volume of ETOH. At day 6, cells and supernatants were harvested and analyzed.

\section{Cell phenotype}


Cell phenotype was analyzed by flow cytometry on a FACSArray bioanalyzer (BD Biosciences, NJ, USA) using FITC-conjugated anti-CD14, -HLA-DR, -CD80, -CD54 and PEconjugated anti-CD1a, -CD86, -CD83 and -CD40 (Beckman Coulter, France).

\section{Mixed leukocyte reactions (MLR)}

T lymphocytes were purified from human peripheral blood as previously described [3]. Primary MLR were conducted in 96-well flat-bottom culture plates. DC treated or not at day 5, were collected at day 6, extensively washed and resuspended in complete RPMI 1640 medium with $10 \%$ fetal calf serum (BioMedia, France). Cells were then co-cultured with $2 \times 10^{5}$ allogeneic T cells in $200 \mu 1$ complete culture medium at $1 / 10 \mathrm{DC} / \mathrm{T}$ cells ratio. After 5 days, co-culture supernatants were harvested.

\section{Cytokine measurement}

Culture supernatants were stored at $-80^{\circ} \mathrm{C}$. Cytokine and chemokine concentration was determined using specific CBA-flex set assays (BD Biosciences), analyzed on a FACSArray instrument (BD Biosciences).

\section{Statistics}

Results are expressed as mean $\pm \mathrm{SE}$ of at least 3 separate experiments. One sided Wilcoxon test was used to compare pairs within groups and $p$ values below 0.05 were considered as statistically significant. 


\section{Results}

\section{Biochemical characterization of the LDL-X particle.}

LDL was treated with hGX-sPLA $2(100-500 \mathrm{nM})$ for $3 \mathrm{~h}$ or $24 \mathrm{~h}$, at $\mathrm{RT}$ or $37^{\circ} \mathrm{C}$ and the biochemical parameters of the resulting phospholipolyzed LDL particle were analyzed. The absence of degradation of the major LDL apolipoprotein, apoB-100, was verified in LDL-X and mock-treated LDL. As shown in figure $1 \mathrm{~A} \& \mathrm{~B}$, the apoB-100 appeared as a single band at the expected molecular weight of $\sim 500 \mathrm{kDa}$ either after Coomassie Blue staining or western blot analysis of LDL-X or mock-treated-LDL, independently of the time of incubation or the concentration of hGX-sPLA 2 used. LDL treatment did not result in oxidative modification of the particle as determined by the lack of production of conjugated dienes (data not shown). Phospholipolysis of LDL by hGX-sPLA 2 resulted in particles with increased electrophoretic mobility on agarose gel as compared to mock-treated LDL $(p<0.05$ for all hGX-sPLA 2 treatments) (Fig.1C). These results indicate a reduced size and an increased negative charge of LDL-X as compared to native LDL, and are in agreement with our previous results showing that LDL treated with $100 \mathrm{nM}$ hGX-sPLA 2 has a smaller diameter than that of mock-treated LDL [12]. These particles were not spontaneously prone to aggregation $[12,14]$. LDL phospholipolysis resulted in an increased production of FFAs and LPC (Fig.1D\&E) which correlated with the concentration of hGX-sPLA 2 used for the treatment. No significant difference was observed in FFA and LPC production between LDL-X treated for $3 \mathrm{~h}$ at $37^{\circ} \mathrm{C}$ or $24 \mathrm{~h}$ at RT. A $24 \mathrm{~h}$ treatment of LDL with $500 \mathrm{nM} \mathrm{hGX-sPLA}$, resulted in the release of $0.86 \pm 0.11 \mathrm{mM}$ LPC, corresponding to the hydrolysis of around $50 \%$ of the initial LDL content in phosphatidylcholine (around $1.4 \mathrm{mM}$ as reported in [14]). From the above results, LDL treatment with $500 \mathrm{nM}$ hGX-sPLA 2 for $24 \mathrm{~h}$ induced a significant increase $(p<0.05)$ in LPC and FFA production as compared to treatment with $100 \mathrm{nM}$, but did not result in a complete hydrolysis of LDL PC. Taken together, these results show that LDL 
phospholipolysis by hGX-sPLA 2 for $3 \mathrm{~h}$ at $37^{\circ} \mathrm{C}$ or for $24 \mathrm{~h}$ at $\mathrm{RT}$ results in a LDL-X particle with similar biochemical properties.

\section{Effect of the hydrolysis products on hGX-sPLA 2 activity.}

The hydrolysis of LDL by hGX-sPLA 2 was not complete even after a $24 \mathrm{~h}$-treatment with $500 \mathrm{nM}$ of hGX-sPLA 2 which meant that either the conformational/structural changes occurring during hydrolysis inhibited the enzyme's access to substrate or that the lipid mediators produced during LDL hydrolysis had a negative feedback on the enzyme's activity. In order to test whether AA or LPC could alter the enzyme activity, we used radioactive PAF as a substrate for hGX-sPLA 2 [21]. As shown in figure 2, hGX-sPLA ${ }_{2}$ activity is inhibited in a concentration dependent manner in the presence of AA whereas the effect of LPC is much weaker. Similarly to AA, other FFAs like linoleic acid also strongly inhibited hGX-sPLA 2 activity (data not shown). These results indicate that lipid mediators released during LDL hydrolysis and mainly the FFAs have a negative impact on hGX-sPLA 2 activity.

\section{Effect of LDL-X on DC maturation.}

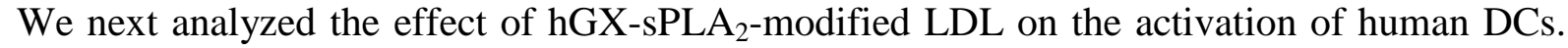
These cells, differentiated from human blood monocytes in a lipoprotein-free culture medium [3], presented a typical phenotype of immature DCs (iDC) at day 6 , defined by high CD1a and low CD14 expression, intermediate levels of major histocompatibility complex class II molecules (HLA-DR) and low levels of several maturation markers such as CD86, CD80, CD83 and CD40 and of the adhesion molecule CD54 (Fig.3A). In the following experiments, LDL was treated with $500 \mathrm{nM}$ hGX-sPLA 2 , for $3 \mathrm{~h}$ at $37^{\circ} \mathrm{C}$ or $24 \mathrm{~h}$ at RT. Lipoproteins were introduced in the cell culture at day 5 for $24 \mathrm{~h}$. The addition of mock treated LDL did not induce any significant modification in the expression of the analyzed surface markers as 
compared to control iDC (Fig.3A). Interestingly, LDL-X induced the up-regulation of mature DC marker expression, especially HLA-DR and CD86 and also to lesser extent CD80, CD83, CD40 and CD54 (Fig.3A). These results indicate that LDL-X induces a phenotypic maturation of DC in vitro. LDL-X obtained after a $24 \mathrm{~h}$-treatment at $\mathrm{RT}$ or $3 \mathrm{~h}$ at $37^{\circ} \mathrm{C}$ had a similar impact on DC phenotypic maturation as shown by the similar up-regulation of CD86 expression (Fig.3B) as well as the expression of other maturation markers (data not shown). In our experimental setting, the up-regulation of CD86 expression is a constant feature of DC maturation and the intensity of its expression correlates with the ability of DC to stimulate T cells $[3,4,9]$. Therefore we used CD86 as a quantitative indicator of DC maturation. The impact of LDL-X on DC maturation was dependent on the dose of hGX-sPLA 2 used for LDL hydrolysis with a strong increase of CD86 expression achieved by LDL treated with 500nM sPLA $_{2}$ (Fig.3C). To avoid introducing chemicals or inhibitors that can interfere with DC maturation, hGX-sPLA 2 was not neutralized after LDL treatment. Therefore the enzyme introduced in DC culture could also hydrolyze membrane DC phospholipids. Since previous results have indicated that different exogenous $\mathrm{SPLA}_{2} \mathrm{~s}$ can induce DC maturation [9-11], we also analyzed the direct impact of hGX-sPLA 2 added to DC culture at the same final concentration as that brought by addition of LDL-X (i.e. 25,10 or $5 \mathrm{nM}$ after dilution of LDL-X to cells). The induction of CD86 expression indicates that the enzyme alone induced DC maturation with almost similar efficiency to that of LDL-X (Fig.3C). Addition of the sPLA $_{2}$ inhibitor LY311727, which does not interfere with either the cytosolic $\mathrm{PLA}_{2}$ or calcium-independent $\mathrm{PLA}_{2}$ activity, abrogated the effect of hGX-sPLA 2 added directly to DCs, whereas it had only a marginal effect on the action of LDL-X on CD86 expression (Fig.3D) and on other DC maturation markers (data not shown). Together, these results indicate that i) the modified LDL-X particle per se and the lipid mediators released during 
LDL phospholipolysis induce DC maturation and ii) hGX-sPLA 2 can also induce DC maturation in the absence of LDL, through hydrolysis of membrane phospholipids.

To characterize the impact of LDL-X and hGX-sPLA ${ }_{2}$ on DC function, we analyzed the secretions of DCs by multiplexed assays for a panel of cytokines and chemokines. The supernatant of control cells receiving only LDL buffer was used to determine basal secretion levels (Fig.4A). LDL treatment of DC slightly increased the secretion of IL-6, IL-8, IP-10 and MIP-1 $\beta$. Compared to LDL, LDL-X induced an increased secretion of MIP-1 $\beta$, MCP-1 and IL-8 (Fig.4A) that failed to be statistically significant, probably because the secretion levels of cytokines were variable depending on blood donors. LDL-X obtained after a $24 \mathrm{~h}$-treatment at RT or a 3 h-treatment at $37^{\circ} \mathrm{C}$ had similar effects (data not shown). DCs that received hGXsPLA $_{2}$ alone secreted higher levels of MIP-1 $\beta$ and MCP-1 than control cells whereas IL- 8 secretion was not increased.

An essential function of mature DCs is their ability to stimulate naïve T cells. Using MLR experiments, we compared the functional properties of DCs treated with LDL-X and LDL versus non treated cells. The results indicate that LDL-X-treated DCs could stimulate T cells and induce IFN $\gamma$ secretion, in contrast to control iDCs and LDL-treated DCs (Fig.4B). This induction of IFN $\gamma$ secretion, associated with low levels of IL-5 and IL-13 in MLR supernatants (data not shown), is characteristic of a Th1 response of T cells.

\section{Lipids generated by hGX-sPLA 2 phospholipolysis of LDL modulate DC maturation.}

The amount of LPC produced from $1 \mathrm{mg} / \mathrm{ml}$ LDL treated with $500 \mathrm{nM}$ hGX-sPLA 2 for $24 \mathrm{~h}$ is $0.86 \mathrm{mM}$ (Fig.1E), thus introducing in DC culture around $40 \mu \mathrm{M}$ LPC with the phospholipolyzed LDL (as described in methods). This dose of LPC is the optimal active dose to stimulate DC maturation in lipoprotein free culture medium (Fig.5A). Even in the presence

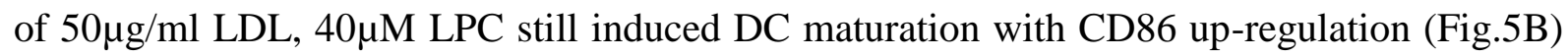


as efficiently as LDL-X (Fig.3B). The diversity of fatty acids esterified at the $s n-1$ and $s n-2$ positions of PC has also to be considered. The major molecular PC species in LDL have been described by others [22]. The hydrolysis of LDL PC by hGX-sPLA 2 results in the production of LPC that contains mainly a palmitic (LPC16:0) or a stearic (LPC18:0) acid esterified at the sn-1 position and low levels of oleic acid (LPC18:1) [15]. Interestingly, both LPC16:0 and LPC18:0 could induce DC maturation in contrast to LPC18:1 (Fig.5C). Several types of fatty acids are released by hGX-sPLA 2 hydrolysis of LDL PC $[15,23]$. We tested the impact of some fatty acids on DC maturation and found that C16:0 and C18:0 induced DC maturation, stimulating CD86 expression whereas C18:1 and AA (C20:4) inhibited LPC-induced DC maturation (Fig.5D-E). These results indicate that both the major molecular species of LPC and the FFA released upon phospholipolysis of LDL may positively or negatively modulate DC maturation depending on their nature. 


\section{Discussion}

In this work, we have shown that phospholipolyzed LDL by hGX-sPLA 2 and the lipid mediators produced during phospholipolysis induce DC maturation, resulting in cells that can stimulate a Th1 response of T cells. LDL was treated with high concentrations of hGX-sPLA 2 in order to achieve the maximum degree of LDL hydrolysis before the 20 -fold dilution in DC culture medium. Although Hanasaki et al. [14], reported that after a 24h-treatment with 50nM hGX-sPLA 2 , PC hydrolysis on LDL was complete, we did not observe a complete PC hydrolysis, using colorimetric assays. We thus hypothesized that either the enzyme could no longer reach the substrate (PC) due to conformational changes on LDL during hydrolysis and/or the lipids released (FFA and LPC) affected the enzyme activity. Using PAF as a substrate, we show here a dose-dependent FFA inhibition of hGX-sPLA 2 activity whereas the effect of LPC was less important. The difference between LPC and FFA effects may be due to their different partition capacities in the PAF micelles used in the assay [21]. As hGX-sPLA 2 preferentially releases AA from cell membranes and lipoproteins, the fact that AA can also inhibit the enzyme activity is quite intriguing but has to be evaluated in a pathophysiological context.

We were unable to detect hGX-sPLA 2 expression in iDCs or in LPS-stimulated mature DCs (data not shown), suggesting that in an in vivo situation such as within the atherosclerotic plaque, the enzyme is likely to be expressed locally by other neighboring cells (for example macrophages) and to activate DCs in a paracrine manner. Different $\mathrm{sPLA}_{2} \mathrm{~S}$ have been detected in normal arterial media and the expression of groups IIA, III, V and $\mathrm{X}$ sPLA 2 increases with atherosclerosis progression $[13,24]$. These enzymes do have different substrate specificities, lipolytic activities and binding properties, therefore likely exerting various effects on cells [20]. Today, increased $\mathrm{SPLA}_{2}$ activity is considered a hallmark of inflammation that can be detected in biological fluids of patients with rheumatoid arthritis, 
acute pancreatitis, sepsis, psoriasis and cancer (for review see [8]). Moreover, circulating $\mathrm{sPLA}_{2}$ activity was shown to predict the risk of coronary events in healthy individuals [25]. However, the specific role of each $\mathrm{SPLA}_{2}$ during pathological conditions remains to be determined in many cases.

DCs exposed to LDL-X or hGX-sPLA 2 acquire a phenotype of mature DCs, mainly characterized by the up-regulation of HLA-DR and CD86 expression, allowing antigen presentation and co-stimulation of $\mathrm{T}$ cells. The cytokine and chemokine secretion profile of LDL-X-treated DCs constitutes a specific signature characterized by the secretion of MIP-1 $\beta$, MCP-1 and IL-8. This secretion profile is very similar to that of LPC-treated DCs (Sup. Fig.1) whereas it strongly differs from the secretion profile of mature DCs stimulated by pathogen associated motifs such as LPS, characterized by massive secretion of IL-12, IL-6, IL-10 and TNF- $\alpha$. The absence of IL-12 secretion was also previously observed for oxLDL- and LPCinduced DC maturation. More generally, the lack of secretion of cytokines such as IL-12 which is mainly driven by NF- $\mathrm{B}$, indicates that TLR4 signaling is probably not involved in the maturation induced by LDL-X or hGX-sPLA 2 . The secretion profile of LDL-X-treated DCs differs slightly from that of hGX-sPLA 2 -treated DCs, for which we did not observe any induction of IL-8 secretion. Our results show that the cytokine secretion profile is the result of the integration of multiple signals delivered by the modified lipoprotein particle and the lipid molecules produced. The amount of LPC produced during LDL phospolipolysis could efficiently induce DC maturation even in the presence of native LDL (Fig.5). Furthermore, the nature of the fatty acids either esterified in LPC or released during PC hydrolysis by sPLA $_{2}$ has also to be taken into account. Both saturated LPC containing palmitate or stearate efficiently trigger DC maturation, stimulating CD86 expression, in contrast to LPC containing oleate. Palmitic and stearic acids alone also induced DC maturation whereas oleic acid could antagonize the effect of saturated LPC and fatty acids. Interestingly, both palmitic and stearic 
acids were shown to exhibit pro-atherogenic properties while oleic acid, a mono-unsaturated species, was anti-atherogenic [26]. Although extracellular AA alone had no significant effect, it could inhibit LPC-induced DC maturation. This effect could be due either to AA by itself or to AA metabolites, which may be rapidly generated in cell culture. Some metabolites of AA such as prostaglandins, thromboxanes and leukotrienes, that are generated in vivo, could modulate DC function [27]. These results indicate that the fatty acid composition of the lipid mediators is essential. Although we cannot exclude that minor species can modulate DC function, the major saturated LPC species produced by PLA 2 hydrolysis of LDL [23] can efficiently induce DC maturation, maybe via a fatty acid dependent mechanism.

Lipids released from lipoproteins during phospholipolysis can be trapped by albumin a major lipid carrier protein. DC experiments were performed in a culture medium containing albumin introduced by the foetal calf serum, therefore, our results show the effect of phospholipolyzed LDL, LPC and FFA in the presence of albumin. To our knowledge, the albumin concentration in normal or atherosclerotic arterial intima is unknown, so it was not possible to adjust more precisely albumin concentration.

DCs exposed to LDL-X engage a functional maturation, acquiring the ability to stimulate $\mathrm{T}$ cells, inducing a Th1 response characterized by IFN $\gamma$ secretion. Although further studies are necessary to establish the impact of LDL-X on the immune system in vivo, our results can be integrated with the current knowledge on phospholipolyzed LDL. In the subendothelial space of inflamed tissues, LDL can be hydrolyzed by hGX-sPLA ${ }_{2}$, generating LPC and FFA which can stimulate cytokine production from endothelial cells, resulting in increased monocyte recruitment [12]. Monocytes entering the tissue will differentiate into macrophages or DCs depending on yet unknown environmental conditions [28]. Phospholipolyzed LDL can contribute to atherogenesis in concert with oxLDL, through the induction of foam cell formation [12], and through the generation of mature DCs able to induce a Th1 response of T 
cells. These cells may either activate neighbouring $\mathrm{T}$ cells in the inflamed tissue or emigrate to draining lymph nodes to subsequently activate $\mathrm{T}$ cells that will be recruited at the site of inflammation. These IFN $\gamma$-secreting Th1 cells can amplify the inflammatory response, by activating endothelial cells and macrophages [29]. Interestingly, stimulation of DC maturation either through LDL-X or through DC membrane hydrolysis by hGX-sPLA 2 could synergize with TLR ligand stimulation, as recently described in macrophages [30]. In conclusion, phospholipolyzed LDL particles per se as well as the lipid mediators produced from lipoprotein or cell plasma membrane hydrolysis by hGX-sPLA 2 are important signals which impact the fine tuning of DC function. 


\section{Acknowledgements}

RA is supported by a grant of Government Francais-DREIC Jerusalem. GL and EN are directors of research at the Centre National de la Recherche Scientifique. This study was supported by the Institut National de la Santé et de la Recherche Médicale (Inserm) and the Agence Nationale pour la Recherche sur le SIDA et les hépatites (ANRS).

The authors declare that there is no conflict of interest.

Authors contributions:

RA, SD and MC: Performed research; analyzed data

GL and CP: Contributed essential reagents and tools, performed experiments

PA, VL and EN: Designed research, analyzed data

SAK and LPC: Designed \& performed experiments, analyzed data, wrote paper 


\section{References}

[1] Hansson GK and Libby P, The immune response in atherosclerosis: a double-edged sword, Nat Rev Immunol, 2006, 6: 508-19.

[2] Niessner A and Weyand CM, Dendritic cells in atherosclerotic disease, Clin Immunol, 2010, 134: 25-32.

[3] Perrin-Cocon L, Coutant F, Agaugue S, et al., Oxidized low-density lipoprotein promotes mature dendritic cell transition from differentiating monocyte, J Immunol, 2001, 167: 3785-91.

[4] Coutant F, Agaugue S, Perrin-Cocon L, et al., Sensing environmental lipids by dendritic cell modulates its function, J Immunol, 2004, 172: 54-60.

[5] Perrin-Cocon L, Agaugue S, Coutant F, et al., Lysophosphatidylcholine is a natural adjuvant that initiates cellular immune responses, Vaccine, 2006, 24: 1254-63.

[6] Perrin-Cocon L, Diaz O, Carreras M, et al., High-density lipoprotein phospholipids interfere with dendritic cell Th1 functional maturation, Immunobiology, 2011, 217: 91-9.

[7] Murakami M, Taketomi Y, Girard C, et al., Emerging roles of secreted phospholipase A2 enzymes: Lessons from transgenic and knockout mice, Biochimie, 2010, 92: 561-82.

[8] Lambeau G and Gelb MH, Biochemistry and physiology of mammalian secreted phospholipases A2, Annu Rev Biochem, 2008, 77: 495-520.

[9] Perrin-Cocon L, Agaugue S, Coutant F, et al., Secretory phospholipase A2 induces dendritic cell maturation, Eur J Immunol, 2004, 34: 2293-302.

[10] Ramoner R, Putz T, Gander H, et al., Dendritic-cell activation by secretory phospholipase A2, Blood, 2005, 105: 3583-7.

[11] Giannattasio G, Fujioka D, Xing W, et al., Group V secretory phospholipase A2 reveals its role in house dust mite-induced allergic pulmonary inflammation by regulation of dendritic cell function, J Immunol, 2010, 185: 4430-8. 
[12] Karabina SA, Brocheriou I, Le Naour G, et al., Atherogenic properties of LDL particles modified by human group X secreted phospholipase A2 on human endothelial cell function, Faseb J, 2006, 20: 2547-9.

[13] Kimura-Matsumoto M, Ishikawa Y, Komiyama K, et al., Expression of secretory phospholipase A2s in human atherosclerosis development, Atherosclerosis, 2008, 196: 81-91. [14] Hanasaki K, Yamada K, Yamamoto S, et al., Potent modification of low density lipoprotein by group $\mathrm{X}$ secretory phospholipase $\mathrm{A} 2$ is linked to macrophage foam cell formation, J Biol Chem, 2002, 277: 29116-24.

[15] Pruzanski W, Lambeau L, Lazdunsky M, et al., Differential hydrolysis of molecular species of lipoprotein phosphatidylcholine by groups IIA, V and X secretory phospholipases A(2), Biochim Biophys Acta, 2005, 1736: 38-50.

[16] Yamamoto K, Isogai $\mathrm{Y}$, Sato $\mathrm{H}$, et al., Secreted phospholipase A2, lipoprotein hydrolysis, and atherosclerosis: integration with lipidomics, Anal Bioanal Chem, 2011, 400: $1829-42$.

[17] Jemel I, Li H, Oslund RC, et al., Group X secreted phospholipase A2 proenzyme is matured by a furin-like protein convertase and release arachidonic acid inside of human HEK293 cells, J Biol Chem, 2011, In Press.

[18] Gora S, Maouche S, Atout R, et al., Phospholipolyzed LDL induces an inflammatory response in endothelial cells through endoplasmic reticulum stress signaling, FASEB J, 2010, 24: 3284-97.

[19] Karabina SA, Gora S, Atout R and Ninio E, Extracellular phospholipases in atherosclerosis, Biochimie, 2010, 92: 594-600.

[20] Bezzine S, Koduri RS, Valentin E, et al., Exogenously added human group X secreted phospholipase $\mathrm{A}(2)$ but not the group IB, IIA, and V enzymes efficiently release arachidonic acid from adherent mammalian cells, J Biol Chem, 2000, 275: 3179-91. 
[21] Gora S, Lambeau G, Bollinger JG, et al., The proinflammatory mediator Platelet Activating Factor is an effective substrate for human group $\mathrm{X}$ secreted phospholipase A2, Biochim Biophys Acta, 2006, 1761: 1093-9.

[22] Wiesner P, Leidl K, Boettcher A, et al., Lipid profiling of FPLC-separated lipoprotein fractions by electrospray ionization tandem mass spectrometry, J Lipid Res, 2009, 50: 574-85. [23] Pruzanski W, Lambeau G, Lazdunski M, et al., Hydrolysis of minor glycerophospholipids of plasma lipoproteins by human group IIA, $\mathrm{V}$ and $\mathrm{X}$ secretory phospholipases A2, Biochim Biophys Acta, 2007, 1771: 5-19.

[24] Oorni K and Kovanen PT, Lipoprotein modification by secretory phospholipase A(2) enzymes contributes to the initiation and progression of atherosclerosis, Curr Opin Lipidol, 2009, 20: 421-7.

[25] Mallat Z, Benessiano J, Simon T, et al., Circulating secretory phospholipase A2 activity and risk of incident coronary events in healthy men and women: the EPIC-Norfolk study, Arterioscler Thromb Vasc Biol, 2007, 27: 1177-83.

[26] Mattson FH and Grundy SM, Comparison of effects of dietary saturated, monounsaturated, and polyunsaturated fatty acids on plasma lipids and lipoproteins in man, $\mathbf{J}$ Lipid Res, 1985, 26: 194-202.

[27] Harizi H and Gualde N, The impact of eicosanoids on the crosstalk between innate and adaptive immunity: the key roles of dendritic cells, Tissue Antigens, 2005, 65: 507-14.

[28] Randolph GJ, Inaba K, Robbiani DF, et al., Differentiation of phagocytic monocytes into lymph node dendritic cells in vivo, Immunity, 1999, 11: 753-61.

[29] Libby P, Ridker PM and Hansson GK, Progress and challenges in translating the biology of atherosclerosis, Nature, 2011, 473: 317-25.

[30] Shridas P, Bailey WM, Talbott KR, et al., Group X Secretory Phospholipase A2 Enhances TLR4 Signaling in Macrophages, J Immunol, 2011, 187: 482-9. 


\section{Figure Legends}

\section{Fig.1. Biochemical characterization of phospholipolyzed LDL (LDL-X)}

LDL $(1 \mathrm{mg} / \mathrm{ml})$ was treated for $3 \mathrm{~h}$ at $37^{\circ} \mathrm{C}(1$ to 4$)$ in the absence (1) or in the presence of 100 , 200, 500nM hGX-PLA 2 (2, 3, 4 respectively), or for 24h at RT (5 to 8), in the absence (5) or in the presence of $100,200,500 \mathrm{nM}$ hGX-PLA $2(6,7,8$ respectively). The proteins of the resulting lipoproteins were then separated by 7\% SDS-PAGE followed by (A) Coomassie Blue staining or (B) Western Blot analysis of apoB-100. Representative of 2 gels with similar results (C) Agarose gel electrophoresis of lipoproteins followed by Sudan Red staining. Representative of 6 gels with similar results. (D) FFA release \& (E) LPC release from the above treated lipoproteins. Results represent the mean $\pm \mathrm{SE}$ of at least 5 experiments in duplicate; ${ }^{*} p<0.05$ when compared to LDL, ${ }^{*} p<0.05$ when compared to $100 \mathrm{nM}$ hGXsPLA $_{2}$-treated LDL.

Fig.2. [ ${ }^{3} \mathrm{H}$-acetyl]PAF hydrolysis by hGX-sPLA $A_{2}$ in the presence of AA and LPC. Recombinant hGX-sPLA 2 was incubated with $\left[{ }^{3} \mathrm{H}\right.$-acetyl]PAF at $37^{\circ} \mathrm{C}$ for 20 min in the presence of increasing concentrations of AA, LPC or both, or equal volume of the vehicle ETOH. Results represent the mean \pm SE of $\left[{ }^{3} \mathrm{H}\right.$-acetyl]PAF hydrolysis from at least 5 experiments performed in duplicate. ${ }^{*} p<0.05$ when compared to ETOH.

\section{Fig.3. Characteristic mature DC phenotype induced by LDL-X.}

The expression of surface markers was analyzed by flow cytometry at day 6 after a 24htreatment with (A) buffer (control iDC), or $50 \mu \mathrm{g} / \mathrm{ml}$ LDL or LDL-X treated with $500 \mathrm{nM}$ hGX-sPLA 2 for $24 \mathrm{~h}$ at RT. (B) CD86 expression of DCs that received 50 $\mu \mathrm{g} / \mathrm{ml} \mathrm{LDL}$ or LDL$\mathrm{X}$ (treated with $500 \mathrm{nM} \mathrm{hGX-sPLA}$ for $24 \mathrm{~h}$ at $\mathrm{RT}$ or for $3 \mathrm{~h}$ at $37^{\circ} \mathrm{C}$ ). (C-D) LDL was treated 


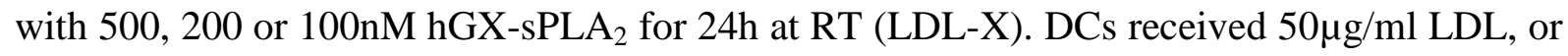
LDL-X or the equivalent amount of hGX-sPLA 2 brought by LDL-X in the cell culture (X 25, 10 or 5). When mentioned, the SPLA $_{2}$ inhibitor LY329722 (LY) was added 15 min before. (BD) The mean fluorescence intensity for CD86 labeling was normalized to control iDCs receiving LDL buffer (fold induction). Results represent the mean \pm SE from 6 (B,C) or 3 (D) independent experiments.

\section{Fig.4. Functional DC maturation induced by LDL-X.}

(A) Cytokine and chemokine secretion profiles. LDL-X was generated by treatment with 500nM hGX-sPLA 2 for $24 \mathrm{~h}$ at RT. DCs received $50 \mu \mathrm{g} / \mathrm{ml} \mathrm{LDL}$ or LDL-X or $25 \mathrm{nM}$ hGXsPLA $_{2}$ alone $(\mathrm{X})$ or control buffer. DC supernatants were collected at day 6 and assayed for cytokines and chemokines secretion. Results represent the mean concentration \pm SE from 3 independent experiments. (B) LDL-X activated DCs can stimulate a Th1 response of T cells. After a $24 \mathrm{~h}$-treatment with $50 \mu \mathrm{g} / \mathrm{ml}$ LDL or LDL-X or buffer, DCs were washed and cocultured in triplicates for 5 days with allogeneic T cells. Mean IFN $\gamma$ secretion in MLR supernatants was normalized to control DCs for each experiment (fold increase). Results represent the mean $\pm \mathrm{SE}$ from 5 independent experiments.

\section{Fig.5. LPC and fatty acids produced by $\mathrm{SPLA} \mathrm{A}_{2}$ activity can modulate DC maturation.}

DCs were treated at day 5 for $24 \mathrm{~h}$ with (A) 20 to $50 \mu \mathrm{M}$ LPC, (B) $40 \mu \mathrm{M}$ LPC in the presence

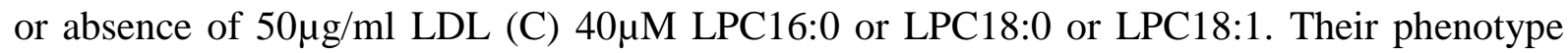
was analyzed at day 6 and CD86 expression is presented as fold induction compared to control iDCs. Results represent the mean \pm SE for 6 independent experiments (A-C). (D-E) DCs were treated at day 5 for $24 \mathrm{~h}$ with $40 \mu \mathrm{M}$ fatty acid with or without $40 \mu \mathrm{M}$ LPC18:0 in the 
presence of $50 \mu \mathrm{g} / \mathrm{ml}$ LDL. DC phenotype was analyzed and CD86 expression is presented as fold induction compared to control iDCs. Results represent the mean \pm SE from 6 experiments. 


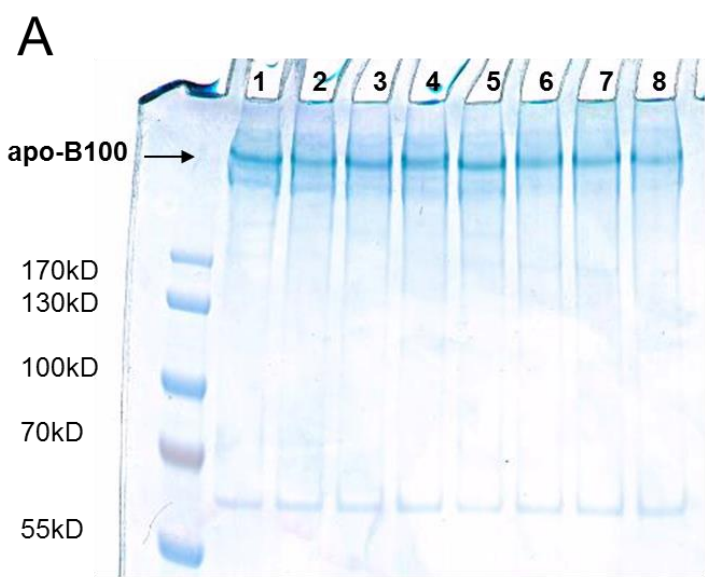

D
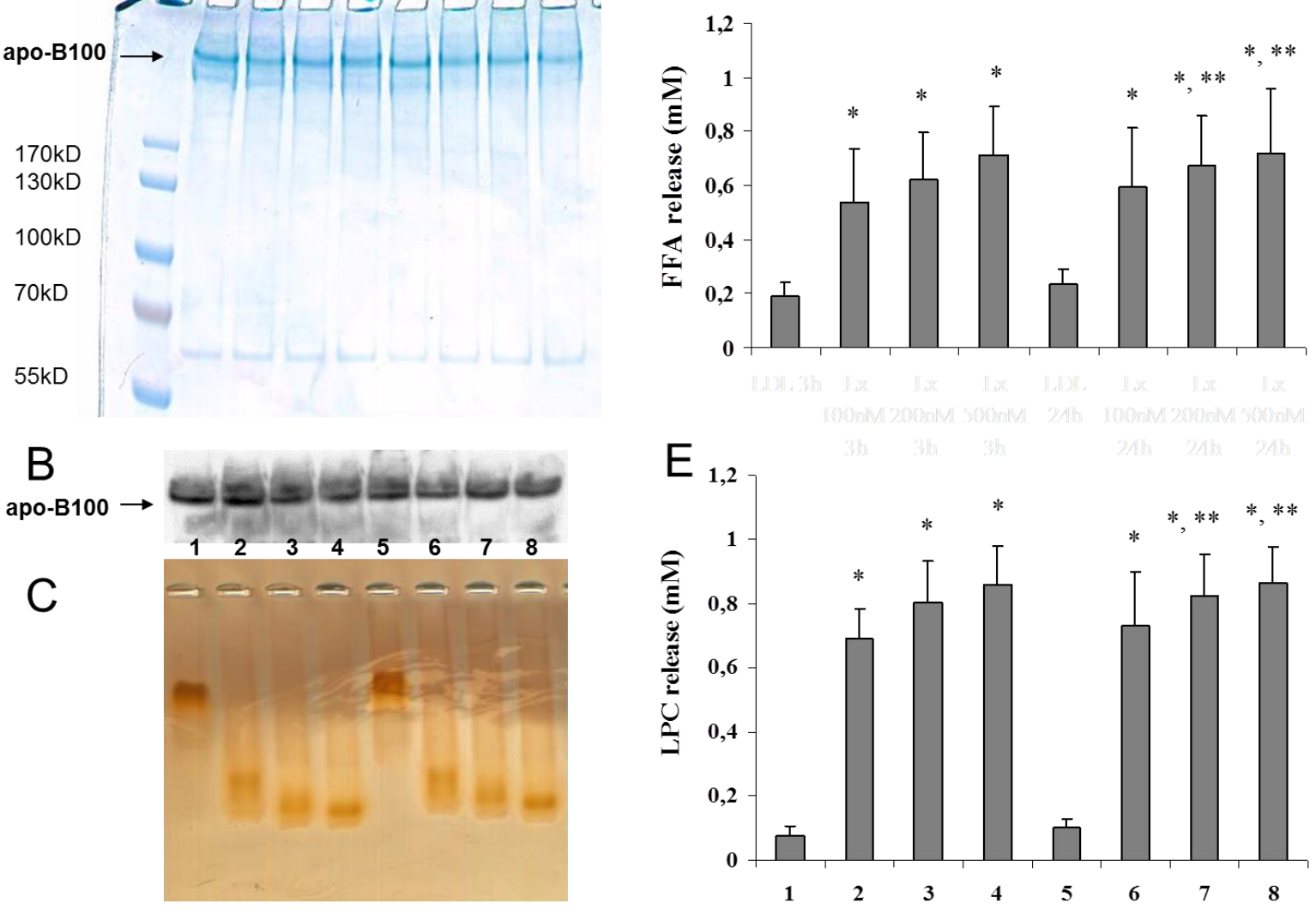

Figure 1 


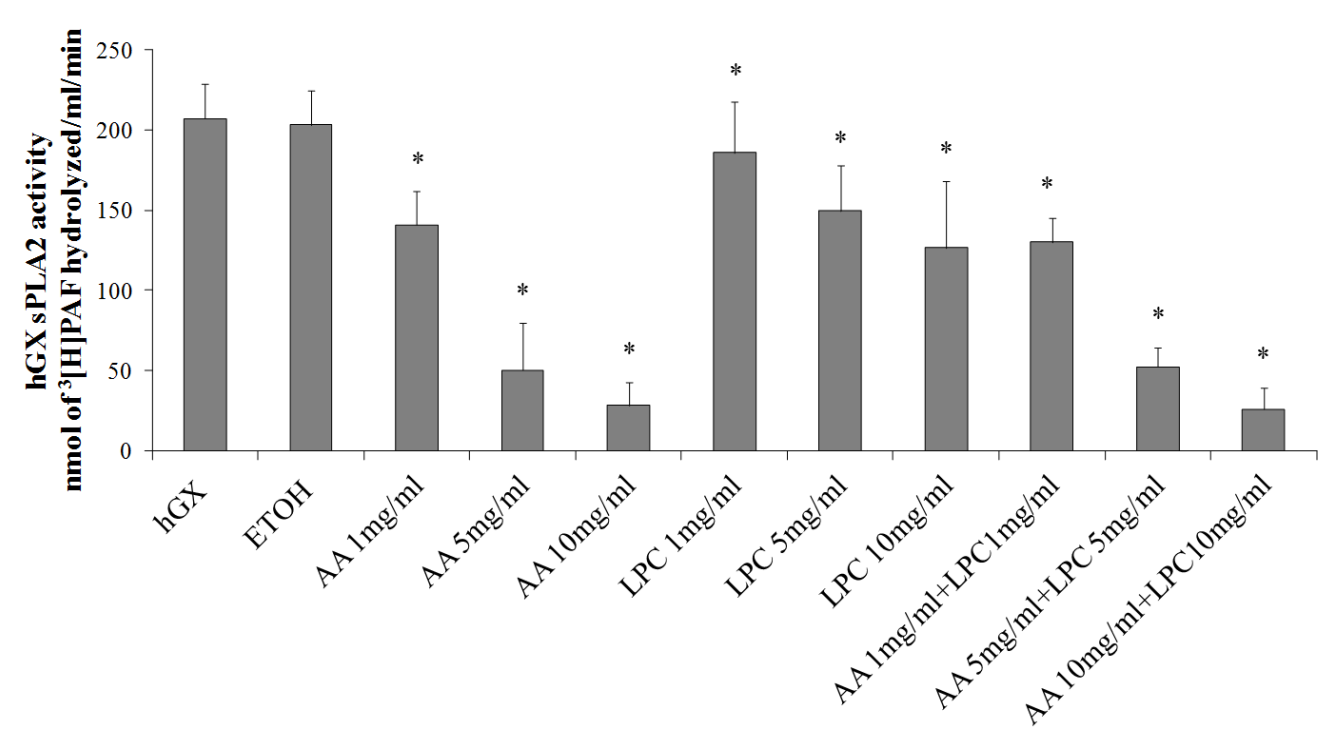

Figure 2 
A

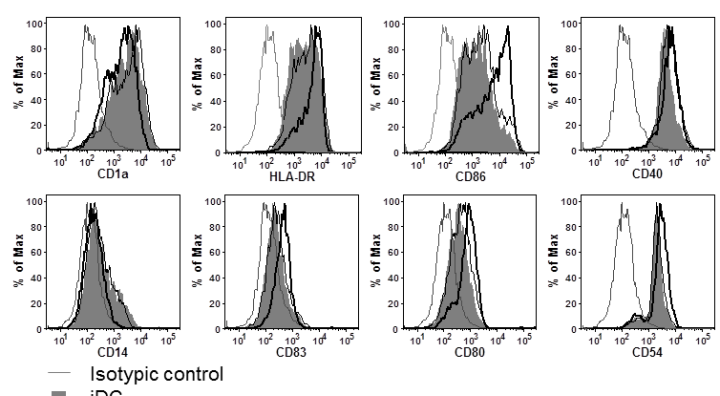

Isotypic control

프 $\mathrm{iDC}$

$\begin{array}{ll}\text { - } & \text { DC + LDL } \\ - & \text { DC + LDL-X }\end{array}$

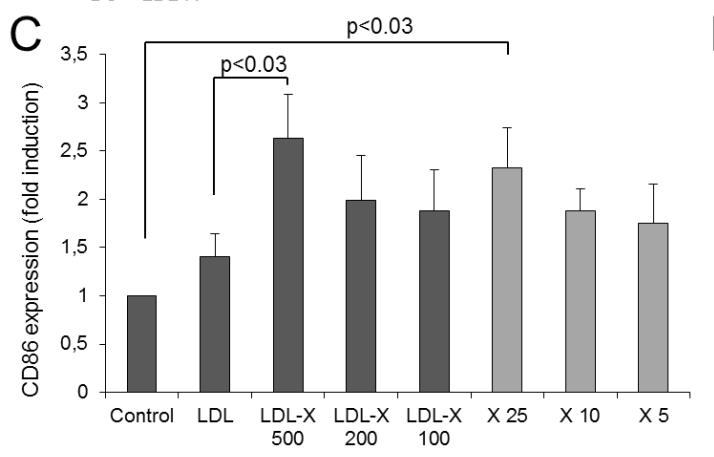

B
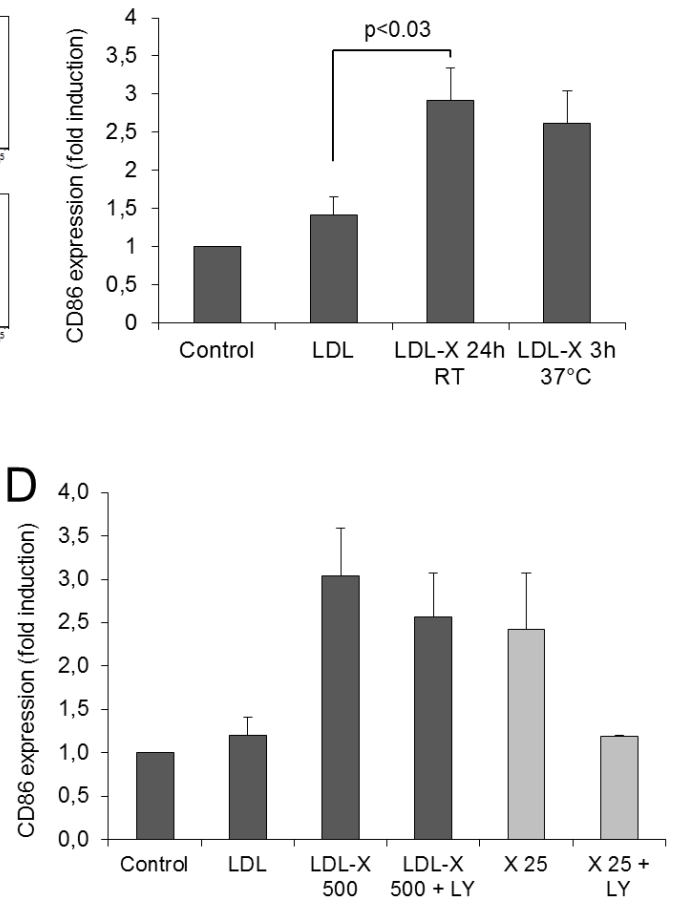

Figure 3 


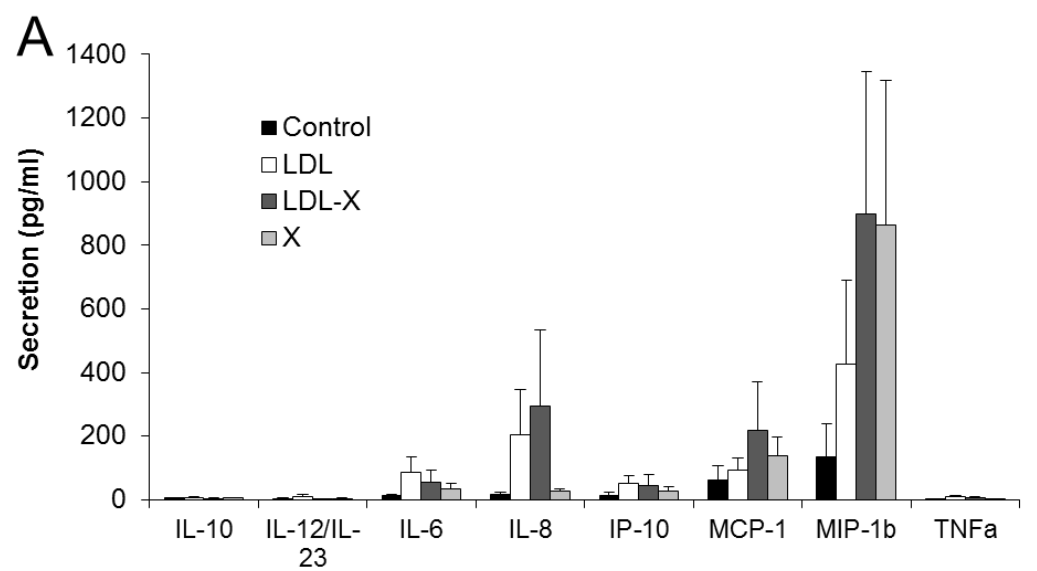

B

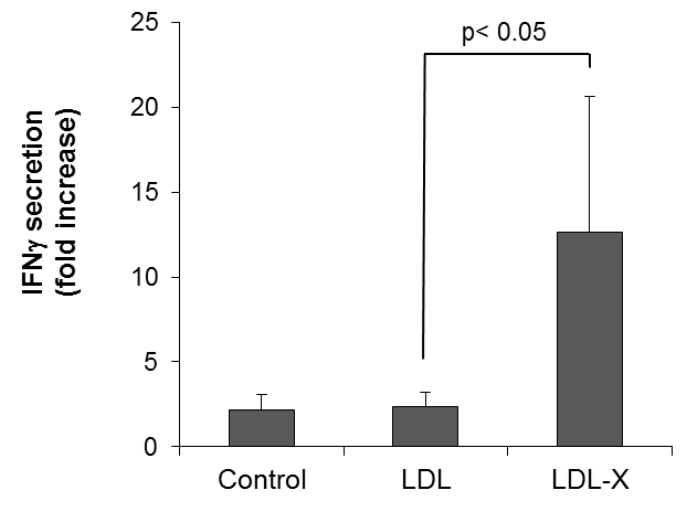

Figure 4 

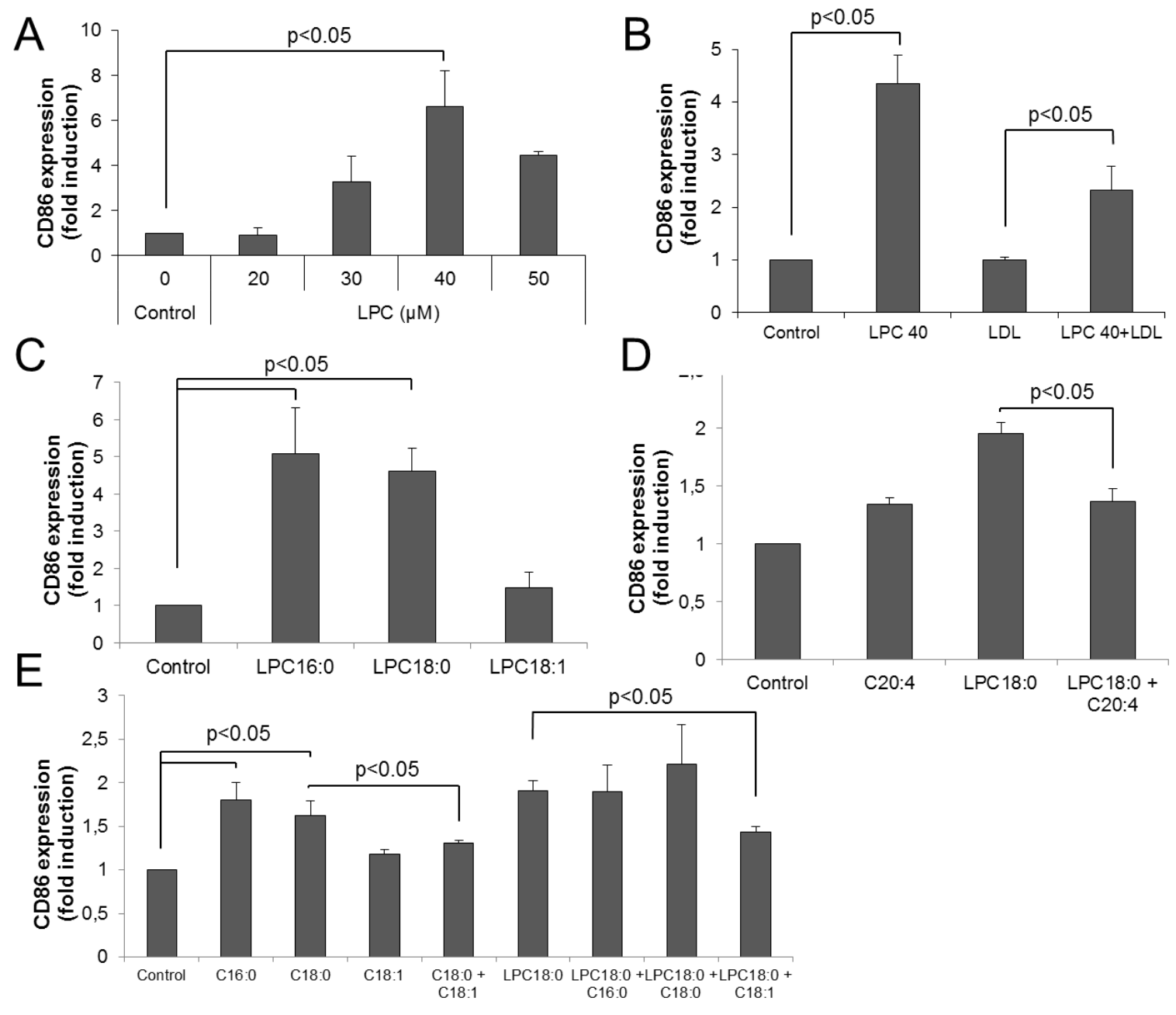

Figure 5 